\title{
BMJ Open Association of vascular access flow with short-term and long-term mortality in chronic haemodialysis patients: a retrospective cohort study
}

\author{
Chung-Kuan Wu, ${ }^{1,2,3}$ Chia-Lin Wu, ${ }^{3,4,5}$ Chia-Hsun Lin, ${ }^{2,6}$ Jyh-Gang Leu, ${ }^{1,2}$ \\ Chew-Teng Kor, ${ }^{7}$ Der-Cherng Tarng ${ }^{3,8,9}$
}

To cite: Wu C-K, Wu C-L, Lin C-H, et al. Association of vascular access flow with shortterm and long-term mortality in chronic haemodialysis patients: a retrospective cohort study. BMJ Open 2017;7:e017035. doi:10.1136/ bmjopen-2017-017035

- Prepublication history and additional material for this paper are available online. To view please visit the journal online (http://dx.doi.org/10.1136/ bmjopen-2017-017035).

C-KW and C-LW contributed equally.

Received 27 March 2017 Revised 27 July 2017 Accepted 4 September 2017

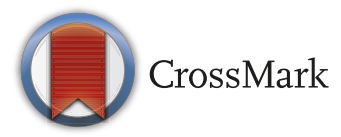

For numbered affiliations see end of article.

Correspondence to Dr Der-Cherng Tarng; dctarng@vghtpe.gov.tw

\section{ABSTRACT}

Objectives To investigate the impact of vascular access flow (Qa) on vascular and all-cause mortality in chronic haemodialysis (HD) patients.

Design Observational cohort study.

Setting Single centre.

Participants Adult chronic HD patients at the HD unit of Shin Kong Wu Ho-Su Memorial Hospital between 1 January 2003 and 31 December 2003 were recruited. Patients were excluded if they had arteriovenous fistula or arteriovenous graft failure within 3 months before the date of Qa measurement, were aged $<18$ years and had Qa levels of $\geq 2000 \mathrm{~mL} / \mathrm{min}$. A total of 378 adult chronic HD patients were eventually enrolled for the study. Interventions The selected patients were evaluated with Qa and cardiac index (Cl). They were divided into four groups according to three Qa cut-off points $(500,1000$ and $1500 \mathrm{~mL} / \mathrm{min}$ ).

Primary and secondary outcome measures Short-term and long-term vascular (cardiovascular or cerebrovascular) and all-cause mortality.

Results Qa was positively correlated with $\mathrm{Cl}(r=0.48$, $\mathrm{p}<0.001$ ). A Qa level of $<1000 \mathrm{~mL} / \mathrm{min}$ was independently associated with 1-year all-cause mortality (adjusted OR, 6.04; $95 \% \mathrm{Cl} 1.64$ to 22.16; $p=0.007$ ). Kaplan-Meier analysis revealed that the cumulative incidence rates of all-cause and vascular mortality were significantly higher in the patients with a Qa level of $<1000 \mathrm{~mL} / \mathrm{min}$ (log-rank test; all $p<0.01$ ). Furthermore, a Qa level of $<1000 \mathrm{~mL} /$ min was independently associated with long-term allcause mortality (adjusted HR, 1.62; 95\% Cl 1.11 to 2.37; $\mathrm{p}=0.013$ ); however, the risk of vascular mortality did not significantly increase after adjustment for confounders. Conclusions $\mathrm{Qa}$ is moderately correlated with cardiac function, and a Qa level of $<1000 \mathrm{~mL} / \mathrm{min}$ is an independent risk factor for both short-term and long-term all-cause mortality in chronic HD patients.

\section{INTRODUCTION}

Vascular access function and patency are crucial for the optimal management of haemodialysis (HD) patients. ${ }^{1}$ Loss of vascular access patency limits HD efficiency, which can result in underdialysis and may increase morbidity

\section{Strengths and limitations of this study}

- The study provides information on the cut-off level of access flow as a predictor of death in chronic haemodialysis (HD) patients, and physicians might be vigilant when the access flow is $<1000 \mathrm{~mL} / \mathrm{min}$ in chronic HD patients.

- A small number of patients may be misclassified because of longitudinal changes in the access flow levels over time.

- Few variables considered to be associated with mortality were unavailable in our registry data based on the retrospective design.

- The patients in our study were recruited from a single medical centre in North Taiwan.

- Changes with the type and location of vascular access in patients during long-term follow-up were not taken into accounts in the cohort study.

and mortality. ${ }^{2}$ The leading causes of loss of vascular access patency for an arteriovenous fistula (AVF) and arteriovenous graft (AVG) are vascular stenosis and thrombosis. ${ }^{3}$ The Kidney Disease Outcomes Quality Initiative (K/DOQI) guidelines recommend vascular access surveillance and monitoring for the early detection of vascular stenosis or thrombosis. ${ }^{45}$ In addition, the $\mathrm{K} / \mathrm{DOQI}$ guidelines recommend vascular interventions when vascular access flow (Qa) becomes $<600 \mathrm{~mL} /$ min in grafts and $<400-500 \mathrm{~mL} / \mathrm{min}$ in fistulae and when Qa decreases by $25 \%$ and to $<1000 \mathrm{~mL} / \mathrm{min}$ over a 4 -month period. ${ }^{6}$ Early treatment of these vascular complications can reduce the progression of vascular stenosis and the rate of thrombosis. ${ }^{7}$

Qa can be affected by many factors including systemic haemodynamics, size and endothelial function of vessels supplying and draining the access and presence of significant vascular stenosis. ${ }^{1}$ Therefore, Qa can be considered a marker of cardiovascular (CV) health in HD patients. ${ }^{8} \mathrm{CV}$ mortality accounts for $45 \%$ of 
all-cause mortality in dialysis patients. ${ }^{9}$ However, the association of Qa with survival in HD patients remains unclear. Low Qa may be associated with underdialysis or poor cardiac status, ${ }^{10}$ whereas a high Qa level $(\geq 2000 \mathrm{~mL} / \mathrm{min})$ has been reported to be associated with left ventricular dilation and high cardiac output $(\mathrm{CO})$ heart failure. ${ }^{11-13}$ Moreover, in HD patients with a Qa level of $<2000 \mathrm{~mL} /$ min, whether an optimal cut-off point for Qa is available for predicting survival remains controversial. ${ }^{8}$

The objectives of the current study were to evaluate the associations among Qa, cardiac function and all-cause or vascular mortality and to provide information regarding the cut-off level for $\mathrm{Qa}$ as a predictor of survival in HD patients.

\section{METHODS}

\section{Study population}

We conducted a retrospective observational cohort study. We retrieved the records of adult patients who underwent chronic HD treatment at the HD unit of Shin Kong Wu Ho-Su Memorial Hospital between 1 January 2003 and 31 December 2003. The observation period was from the date of Qa measurement until the end of 2014. Studies have reported that extremely high Qa levels $(\geq 2000 \mathrm{~mL} /$ $\min )$ contribute to adverse cardiac outcomes. ${ }^{11-13}$ Moreover, there were only 16 patients with extremely high Qa levels in our HD unit. Therefore, we excluded patients who had AVF or AVG failure within 3 months before the date of Qa measurement to ensure no obvious Qa change, were aged $<18$ years and had Qa levels of $\geq 2000 \mathrm{~mL} / \mathrm{min}$. A total of 378 adult chronic HD patients were eventually enrolled for the study. They were divided into four groups according to three Qa cut-off points $(500,1000$ and $1500 \mathrm{~mL} / \mathrm{min}$ ). The study was carried out in strict accordance with guidelines for research involving human subjects developed by the Taiwan Ministry of Health and Welfare. This study was approved by the Institutional Review Board of Shin Kong Wu Ho-Su Memorial Hospital, and the Institutional Review Board waived the requirement for obtaining informed consent in this retrospective study.

\section{History collection and laboratory data}

Demographic and baseline clinical data were obtained from the patients' medical records during the entry in the study; the data included age; sex; comorbid disease history; blood pressure; cardiac index (CI); end-stage renal disease (ESRD) causes; vascular access type; HD vintage; erythropoiesis-stimulating agent (ESA) dosage; lipid and iron profiles; haemoglobin, serum albumin, intact parathyroid hormone (iPTH), sodium, potassium, ionised calcium and phosphate levels; and HD efficiency $(\mathrm{Kt} / \mathrm{V})$. Blood samples were collected after a fasting period of at least 8 hours before each HD session. Kt/V was determined according to the procedure described by Gotch and Sargent. ${ }^{14}$

\section{Measurement of Qa and $\mathrm{Cl}$}

Access flow was measured in all patients by using the Transonic HD01 ${ }^{\text {plus }}$ device (Transonic, Ithaca, New York, USA) on the basis of the method of Krivitski. ${ }^{15}$ The method was regarded as a reference method for access flow evaluation because of high reproducibility and accuracy. ${ }^{16-18}$ In brief, Qa was measured by the indicator-dilution technique where ultrasound was used to detect the difference in saline concentration between the venous and arterial limbs of the dialysis circuit after a bolus of saline was delivered into the venous line when arterial and venous lines were in reversed configuration. Qa was measured at least twice in each session, and the mean of all levels measured at entry of the study was regarded as baseline Qa for analysis. In addition, CO was measured through a similar ultrasound dilution technique using the same machine. CI was calculated by dividing $\mathrm{CO}$ with the body surface area.

\section{Study outcomes}

The primary outcomes were all-cause and vascular (CV or cerebrovascular) mortality. In addition, we evaluated the association of Qa with cardiac function.

\section{Statistical analyses}

The results are presented as a number (percentage) for categorical data, mean (SD) for normally distributed continuous data and median (IQR) for non-normally distributed continuous data. Missing values for each variable were less than $2 \%$ in the whole cohort. The Kolmogorov-Smirnov test was used to test the normal distribution for each variable. Non-normally distributed variables were either log-transformed (eg, CI) for the correlation analysis or analysed using non-parametric tests. Multiple comparisons among the four groups were performed using the Kruskal-Wallis test for continuous variables or the Pearson $\chi^{2}$ test for categorical variables or the Fisher's exact test for categorical variables if the number was less than five in each cell. Correlations were analysed using the Pearson correlation test. The patients were followed from the date of Qa measurement until death. Moreover, the patients were followed until loss to follow-up, kidney transplantation or end of the study period. We also generated a time-dependent receiver operating characteristic (ROC) curve for censored survival data to determine the optimal cut-off level for Qa to predict mortality in our HD patients. ${ }^{19}$ We performed univariate logistic regression analysis to calculate the crude OR of all-cause mortality within 1 year and its association with the Qa groups. Subsequently, we performed multivariate logistic regression analysis to calculate the adjusted OR for potential confounders. We calculated the cumulative incidences of all-cause and vascular mortality during the follow-up period by using the Kaplan-Meier method and compared these between the higher Qa and lower Qa groups by using the log-rank test. The proportional hazards assumption was confirmed by a $\log (-\log$ (survival)) versus log of survival time plot. Univariate Cox's 
proportional hazard models were used to estimate the relative risk (crude (HR)) of all-cause mortality among the Qa groups during the follow-up period, followed by multivariate Cox's analysis to estimate the aHRs. Covariates with a variance inflation factor of $>5$ were considered to exhibit multicollinearity and were excluded from the multivariate regression analysis. Because the risk of $\mathrm{CV}$ or cerebrovascular mortality could be confounded by the competing risk of other death causes, we generated a competing risk model by specifying the cause-specific HRs on the basis of Cox's proportional hazard regression. Furthermore, the interactions of Qa among clinical characteristics were examined using likelihood ratio tests. All statistical analyses were performed using SAS software (V.9.4) and R software (V.3.2.3; R Foundation for Statistical Computing). Two-sided $p$ values of $<0.05$ were considered statistically significant.

\section{RESULTS}

\section{Baseline characteristics}

We retrieved the records of 378 adult patients with ESRD who received regular HD sessions with a mean dialysis vintage of $64.4 \pm 34.3$ months. All patients underwent at least 9 months of HD treatment. The mean age of the cohort was $58.7 \pm 13.2$ years, and the mean follow-up period was $78.1 \pm 50.3$ months. During the follow-up period, all-cause mortality events were determined in these patients including fatal CV events $(n=79)$, fatal cerebrovascular events $(n=14)$, infectious diseases $(n=36)$, malignancies $(\mathrm{n}=10)$, chronic obstructive lung diseases or other lung diseases $(n=10)$, gastrointestinal bleeding $(n=3)$, liver cirrhosis $(n=2)$, others $(n=29)$ and unknown $(n=7)$. Fifty patients dropped out of the study because of transfers to other hospitals, transplantation or treatment modality change. Table 1 lists the baseline characteristics of the patients in terms of Qa. All the patients were divided into four subgroups on the basis of their Qa levels $(<500,500-999.9,1000-1499.9$ and $1500-1999.9 \mathrm{~mL} /$ $\min )$. The prevalence rates of the Qa level of $<500$, 500-999.9, 1000-1499.9 and $1500-1999.9 \mathrm{~mL} / \mathrm{min}$ were $14.0 \%$ (53/378), $45.5 \%$ (172/378), $28.3 \%$ (107/378) and $12.2 \%$ (46/378), respectively. Age, presence of diabetes mellitus, cerebrovascular accident (CVA), CI, ESRD causes, serum albumin, potassium, phosphate, and iPTH levels, Kt/V urea and all-cause mortality rates during the follow-up period (all $\mathrm{p}<0.05$ ) significantly differed among the four subgroups.

\section{Correlation between Qa and $\mathrm{Cl}$}

A Pearson correlation analysis revealed that Qa was significantly and positively associated with a log-transformed CI among all patients $(r=0.48 ; \mathrm{p}<0.001$; figure 1$)$.

\section{Association of Qa with 1-year all-cause mortality}

A Qa level of $<1000 \mathrm{~mL} / \mathrm{min}$ was a significant predictor of all-cause mortality within 1 year after the Qa measurement. Compared with the patients with a Qa level of $\geq 1000 \mathrm{~mL} /$ min, the OR for all-cause mortality within 1 year after the Qa measurement was 3.41 (95\% CI 1.37 to 8.47; $\mathrm{p}=0.008$ ) in patients with a Qa level of $<1000 \mathrm{~mL} / \mathrm{min}$. Moreover, in the multivariate analysis, the OR for all-cause mortality within 1 year after the Qa measurement was 6.04 (95\% CI 1.64 to $22.16 ; \mathrm{p}=0.007$; table 2 ) in patients with a $\mathrm{Qa}$ level of $<1000 \mathrm{~mL} / \mathrm{min}$, after adjustment for potential confounders. Therefore, a lower Qa level $(<1000 \mathrm{~mL} /$ min) was a significant and independent predictor of 1-year all-cause mortality.

\section{Association of Qa with long-term all-cause mortality}

During the 12-year follow-up period, 190 deaths were recorded. The incidence of all-cause mortality was higher in the lower Qa groups (500-999.9 and $<500 \mathrm{~mL} / \mathrm{min}$ ) than in the higher Qa groups (1000-1499.9 and 1500$1999.9 \mathrm{~mL} / \mathrm{min} ; \mathrm{p}=0.001$; figure 2 ). Therefore, a Qa level of $<1000 \mathrm{~mL} / \mathrm{min}$ seems to be a cut-off level for poor prognosis. Furthermore, we identified the most discriminatory value of baseline Qa for all-cause mortality by using time-dependent ROC curve analysis. In agreement with the Kaplan-Meier curves, the optimal cut-off level of Qa for all-cause mortality was $1020 \mathrm{~mL} / \mathrm{min}$ (online supplementary figure S1). Compared with the patients with a Qa level of $\geq 1000 \mathrm{~mL} / \mathrm{min}$, the HR for all-cause mortality was 1.83 (95\% CI 1.35 to 2.49$)$ in patients with a Qa level of $<1000 \mathrm{~mL} / \mathrm{min}$. A Qa level of $<1000 \mathrm{~mL} / \mathrm{min}$ was a significant risk factor for all-cause mortality $(\mathrm{p}<0.001)$. After adjustment for potential confounders, a Qa level of $<1000 \mathrm{~mL} / \mathrm{min}$ remained a significant risk factor for all-cause mortality (adjusted HR (aHR) 1.62; 95\% CI 1.11 to 2.37; $\mathrm{p}=0.013$; table 3 ). Moreover, the aHRs were significantly lower in the higher Qa groups (1000-1499.9 and $1500-1999.9 \mathrm{~mL} / \mathrm{min}$ ) than in the lower Qa group (500-999.9 mL/min; aHRs 0.60 and 0.51 , respectively; both $\mathrm{p}<0.05$; table 4 ). Therefore, a Qa level of $<1000 \mathrm{~mL} /$ min was a significant and independent risk factor for all-cause mortality. In subgroup analyses, higher aHRs for all-cause mortality were observed only in patients who were aged $<65$ years; were men; had diabetes mellitus, hypertension and coronary artery disease $(\mathrm{CAD})$; had a CI of $>4 \mathrm{~L} / \mathrm{min} / \mathrm{m}^{2}$; did not have CVA; and had fistulae (all $\mathrm{p}<0.05$; figure 3 ). In addition, Qa significantly interacted with age, sex and CI (all $\mathrm{p}$ for interactions $<0.05$; figure 3).

\section{Association of Qa with vascular mortality}

We determined 79 fatal CV events and 14 fatal cerebrovascular events during the follow-up period. KaplanMeier analysis revealed a higher incidence of vascular mortality (CV or cerebrovascular mortality) in the lower Qa group $(<1000 \mathrm{~mL} / \mathrm{min})$ than in the higher Qa group ( $\geq 1000 \mathrm{~mL} / \mathrm{min} ; \mathrm{p}=0.008$; figure 4$)$. The cHR for vascular mortality was significantly higher in the patients with a lower Qa level than in those with a higher Qa level (1.79; $95 \%$ CI 1.16 to 2.77 ; $\mathrm{p}=0.009$; table 3$)$. However, after adjustment for potential confounders, the HR for vascular mortality became borderline significant in the 
Table 1 Characteristics of patients according to access blood flow status

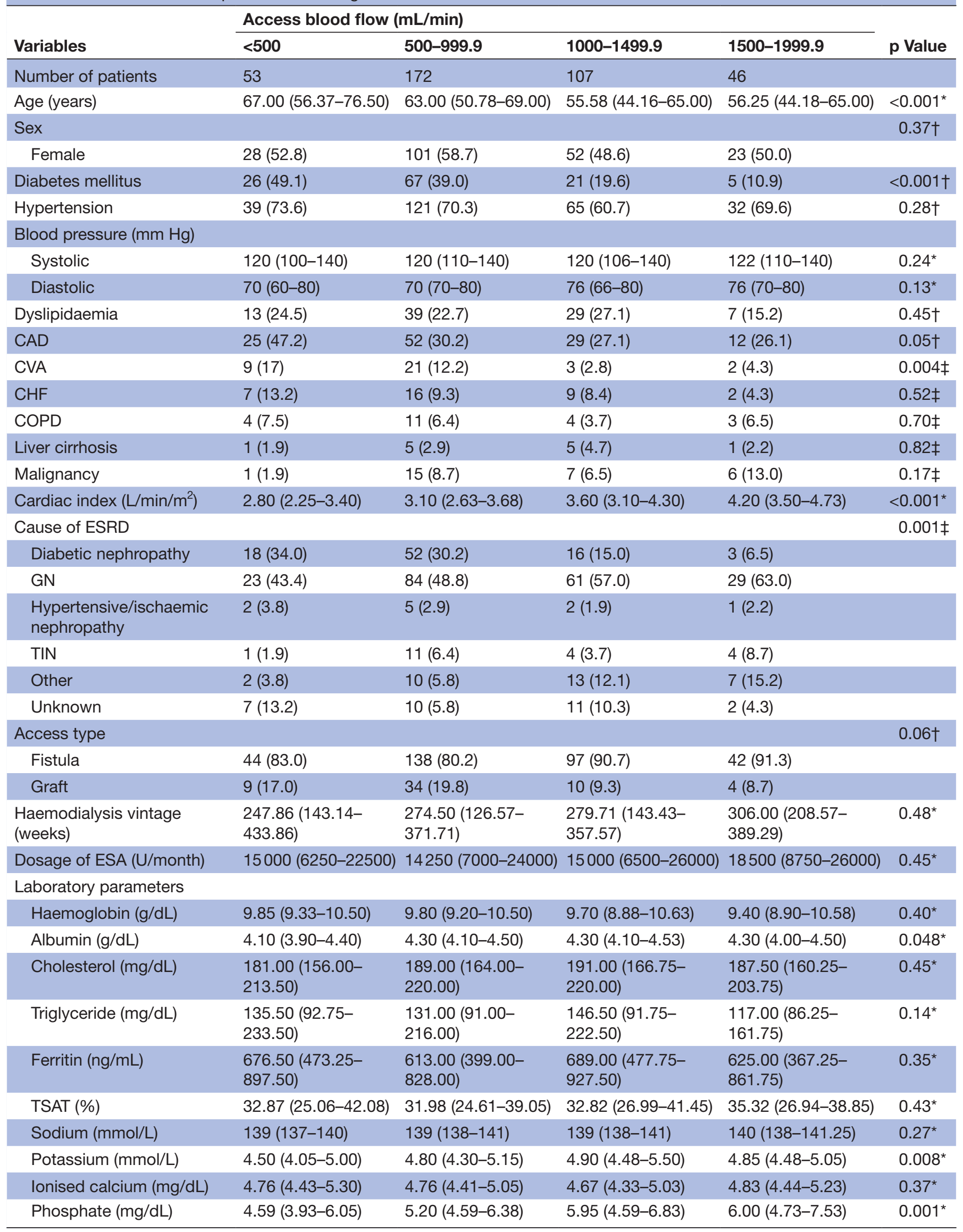


Table 1 Continued

\begin{tabular}{|c|c|c|c|c|c|}
\hline \multirow[b]{2}{*}{ Variables } & \multicolumn{4}{|c|}{ Access blood flow (mL/min) } & \multirow[b]{2}{*}{ p Value } \\
\hline & $<500$ & 500-999.9 & $1000-1499.9$ & $1500-1999.9$ & \\
\hline iPTH (pg/mL) & $\begin{array}{l}43.62(11.82- \\
121.47)\end{array}$ & $\begin{array}{l}73.87(25.28- \\
184.34)\end{array}$ & $\begin{array}{l}90.53(36.10- \\
177.88)\end{array}$ & $\begin{array}{l}68.00(22.00- \\
236.50)\end{array}$ & $0.03^{*}$ \\
\hline $\mathrm{Kt} / \mathrm{V}$ urea & $1.36(1.27-1.60)$ & $1.41(1.29-1.55)$ & $1.34(1.23-1.48)$ & $1.40(1.27-1.53)$ & $0.03^{*}$ \\
\hline \multicolumn{6}{|c|}{ Outcomes during follow-up period } \\
\hline
\end{tabular}

Data are expressed as $\mathrm{n}(\%)$ for categorical data and as medians (interquartile ranges) for continuous data.

${ }^{*}$ Kruskal-Wallis test.

†Pearson $\chi^{2}$ test.

‡Fisher's exact test.

$\S$ Vascular mortality was defined by a composite of cardiovascular or cerebrovascular death.

CAD, coronary artery disease; CHF, congestive heart failure; COPD, chronic obstructive pulmonary disease; CVA, cerebrovascular accident;

ESA, erythropoiesis-stimulating agent; ESRD, end-stage renal disease; GN, glomerulonephritis; iPTH, intact parathyroid hormone; TIN,

tubulointerstitial nephritis.; TSAT, transferrin saturation.

patients with a lower Qa level compared with those with a higher Qa level (aHR 1.69; 95\% CI 0.98 to 2.9; $\mathrm{p}=0.059$; table 3). Therefore, a lower Qa level was a significant but not an independent risk factor for vascular mortality in HD patients.

\section{Sensitivity analyses for misclassification}

Because even a small difference in cut-off levels for higher or lower Qa groups can result in misclassification, we also conducted survival analyses for all-cause and vascular mortality by using $1020 \mathrm{~mL} / \mathrm{min}$ as a cut-off point (online supplementary table S1). The results of sensitivity analyses are consistent with the main findings in table 3 . In addition, vascular interventions in failing accesses may lead to misclassification and affect the outcomes of our study patients. Among patients with a $\mathrm{Qa}<500 \mathrm{~mL} / \mathrm{min}, 21$ of 53

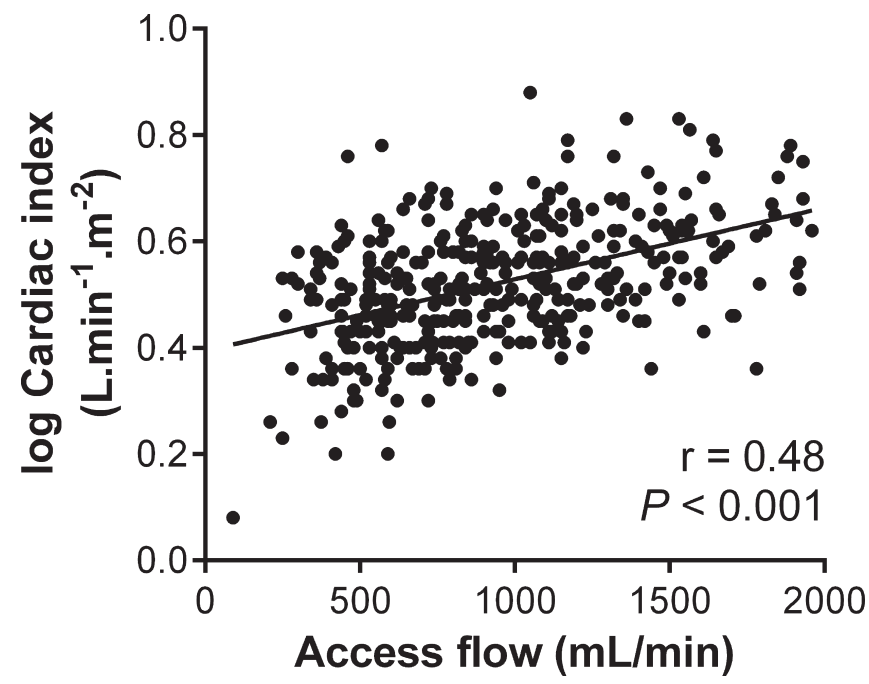

Figure 1 Access flow positively correlated with heart function. Pearson's correlation analysis revealed a linear correlation between access flow rate and log-transformed cardiac index among all study patients. Blood pressure (mm Hg).
(39.6\%) patients underwent vascular interventions during the follow-up period. The medians (IQRs) of Qa were 410 (320-440) $\mathrm{mL} / \mathrm{min}$ before intervention and 530 (410$757.5) \mathrm{mL} / \mathrm{min}$ after intervention. Vascular interventions improved $\mathrm{Qa}$ in these patients $(\mathrm{p}=0.003$, Wilcoxon signedrank test) and $12(22.6 \%)$ patients moved to another group after intervention. However, vascular interventions did not have a significant impact on misclassification regarding the threshold of low $\mathrm{Qa}=1000 \mathrm{~mL} / \mathrm{min}$. Considering patients with a $\mathrm{Qa}<500 \mathrm{~mL} / \mathrm{min}$ who were reclassified into another class after vascular intervention, the results are in agreement with the main findings of this study (online supplementary table S2 and figure S2). Lastly, proportions of all-cause and vascular mortality in patients stratified by a Qa level of $1000 \mathrm{~mL} / \mathrm{min}$ were in consistent with those in table 1 (online supplementary table S3).

Table 2 Logistic regression analysis for the risk of all-cause mortality among the access flow groups within 1 year

\begin{tabular}{lcl}
\hline & \multicolumn{1}{c}{ All-cause mortality } \\
\cline { 2 - 3 } Analysis & OR $(95 \% \mathrm{Cl})$ & p Value \\
\hline Univariate & & \\
Access flow $\geq 1000 \mathrm{~mL} / \mathrm{min}$ & 1 (reference) & - \\
Access flow $<1000 \mathrm{~mL} / \mathrm{min}$ & 3.41 (1.37 to 8.47$)$ & 0.008 \\
Multivariate & & \\
Access flow $\geq 1000 \mathrm{~mL} / \mathrm{min}$ & 1 (reference) & - \\
\hline Access flow $<1000 \mathrm{~mL} / \mathrm{min}$ & 6.04 (1.64 to 22.16) & 0.007 \\
\hline
\end{tabular}

*Adjusted for age; sex; diabetes mellitus; CAD; CHF; CVA; COPD; liver cirrhosis; malignancy; systolic blood pressure; diastolic blood pressure; haemodialysis vintage; vascular access type; ESRD causes; cardiac index; haemoglobin, albumin, cholesterol, ferritin, sodium, potassium, ionised calcium, phosphate and parathyroid hormone levels; $\mathrm{Kt} / \mathrm{V}$; and ESA dosage.

$\mathrm{CAD}$, coronary artery disease; $\mathrm{CHF}$, congestive heart failure; COPD, chronic obstructive pulmonary disease; CVA, cerebrovascular accident; ESA, erythropoiesis-stimulating agent; ESRD, end-stage renal disease. 


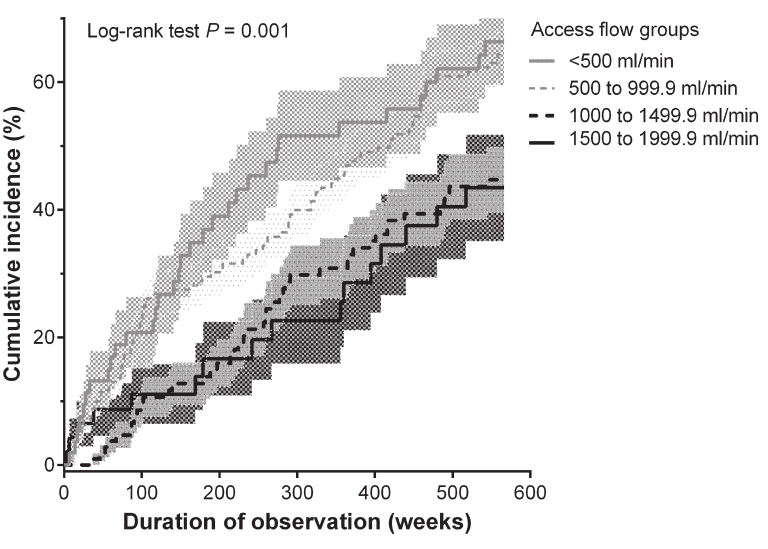

$\begin{array}{lllllll}\text { Qa }<500 & 53 & 41 & 30 & 24 & 23 & 19\end{array}$

$\begin{array}{lllllll}\text { Qa 500-999.9 } & 172 & 120 & 102 & 87 & 74 & 57\end{array}$

Qa 1000-1499.9 $107 \quad 89 \quad 80 \quad 67 \quad 61 \quad 54$

Qa 1500-1999.9 $46 \quad 35 \quad 29 \quad 27 \quad 24 \quad 21$

Figure 2 Cumulative incidences (with standard errors) of all-cause mortality among the patients in different access flow (Qa) groups. Kaplan-Meier analysis revealed that the incidence rate of all-cause mortality was significantly higher in the lower Qa groups (500-999.9 and $<500 \mathrm{~mL} / \mathrm{min}$ ) than in the higher Qa groups (1000-1499.9 and 1500-1999.9 mL/min) during the follow-up period (log-rank test; $p=0.001$ ).

\section{DISCUSSION}

In this observational cohort study, we report that a lower Qa level $(<1000 \mathrm{~mL} / \mathrm{min})$ was associated with an increased risk of short-term and long-term all-cause mortality in chronic HD patients. These chronic HD patients with a lower Qa level were older and were associated with a higher proportion of diabetes mellitus and CVA, lower CI and lower serum albumin, potassium, phosphate and iPTH levels. As reported in the US Renal Data System 2009 annual data report, survival decreased with age, and HD patients with diabetes had poorer survival at 10 years compared with those without diabetes. ${ }^{20}$ Hypoalbuminaemia, hypokalaemia, hypophosphataemia and
Table 4 Multivariate Cox proportional hazards analysis for relative risk of all-cause mortality calculated for four access flow groups during the follow-up period

\begin{tabular}{|c|c|c|}
\hline \multirow[b]{2}{*}{ Analysis } & \multicolumn{2}{|l|}{ All-cause death } \\
\hline & Adjusted HR* $(95 \% \mathrm{Cl})$ & p Value \\
\hline \multicolumn{3}{|c|}{ Access flow (mL/min) } \\
\hline$<500$ & $0.72(0.46$ to 1.12$)$ & 0.14 \\
\hline 500-999.9 & 1 (reference) & - \\
\hline $1000-1499.9$ & $0.60(0.40$ to 0.92$)$ & 0.018 \\
\hline $1500-1999.9$ & $0.51(0.27$ to 0.93$)$ & 0.029 \\
\hline
\end{tabular}

*Adjusted for age; sex; diabetes mellitus; CAD; CHF; CVA; COPD; liver cirrhosis; malignancy; systolic blood pressure; diastolic blood pressure; haemodialysis vintage; vascular access type; ESRD cause; cardiac index; haemoglobin, albumin, cholesterol, ferritin, sodium, potassium, ionised calcium, phosphate and parathyroid hormone levels; Kt/V; and ESA dosage.

$\mathrm{CAD}$, coronary artery disease; $\mathrm{CHF}$, congestive heart failure; COPD, chronic obstructive pulmonary disease; CVA, cerebrovascular accident; ESA, erythropoiesis-stimulating agents; ESRD, end-stage renal disease.

low parathyroid hormone levels are associated with malnutrition and increased mortality rates in chronic HD patients. ${ }^{21-24}$ These data imply that the increased risk of mortality predicted by lower Qa levels may be attributed to the above-mentioned confounders. However, after adjustment for potential cofounders, the patients with lower Qa levels $(<1000 \mathrm{~mL} / \mathrm{min})$ still had a significantly higher risk of short-term and longterm all-cause mortality.

To determine the optimal cut-off level of Qa for all-cause mortality, we performed the time-dependent ROC analysis. The calculated optimal cut-off level $(1020 \mathrm{~mL} / \mathrm{min})$ is in agreement with that calculated in the Kaplan-Meier analysis $(1000 \mathrm{~mL} / \mathrm{min})$. This small difference in cut-off levels for classifying the higher or lower $\mathrm{Qa}$ groups did not engender

Table 3 Cox proportional hazard analysis for the relative risk of all-cause and vascular mortality among the access flow groups during the follow-up period

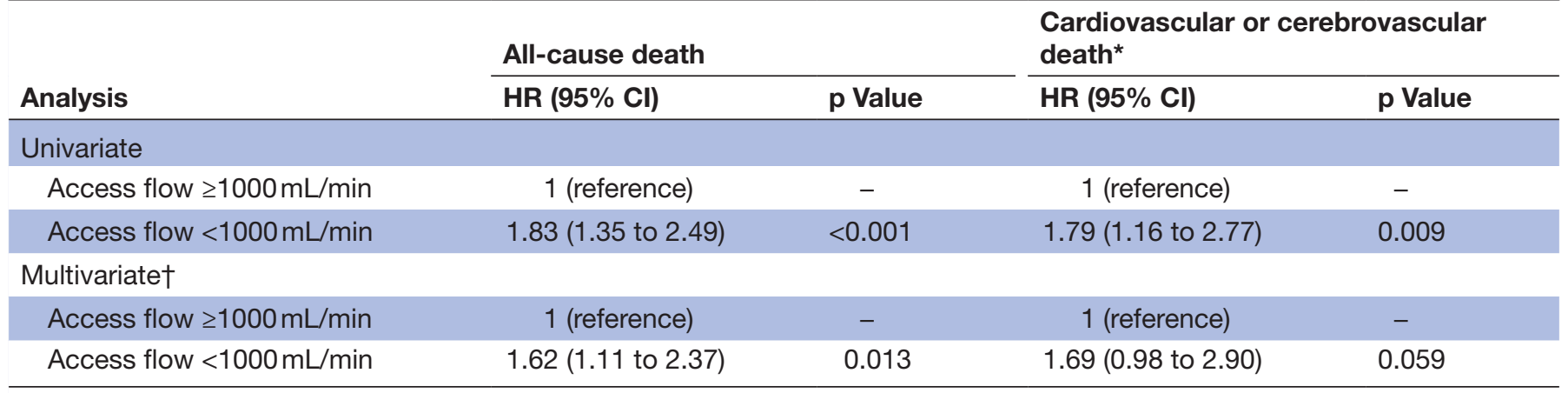

${ }^{*}$ Competing risks with cause-specific hazards.

†Adjusted for age; sex; diabetes mellitus; CAD; CHF; CVA; COPD; liver cirrhosis; malignancy; systolic blood pressure; diastolic blood pressure; haemodialysis vintage; vascular access type; ESRD cause; cardiac index; haemoglobin, albumin, cholesterol, ferritin, sodium, potassium, ionised calcium, phosphate and parathyroid hormone levels; Kt/V; and ESA dosage.

$\mathrm{CAD}$, coronary artery disease; CHF, congestive heart failure; COPD, chronic obstructive pulmonary disease; CVA, cerebrovascular accident; ESA, erythropoiesis-stimulating agent; ESRD, end-stage renal disease. 


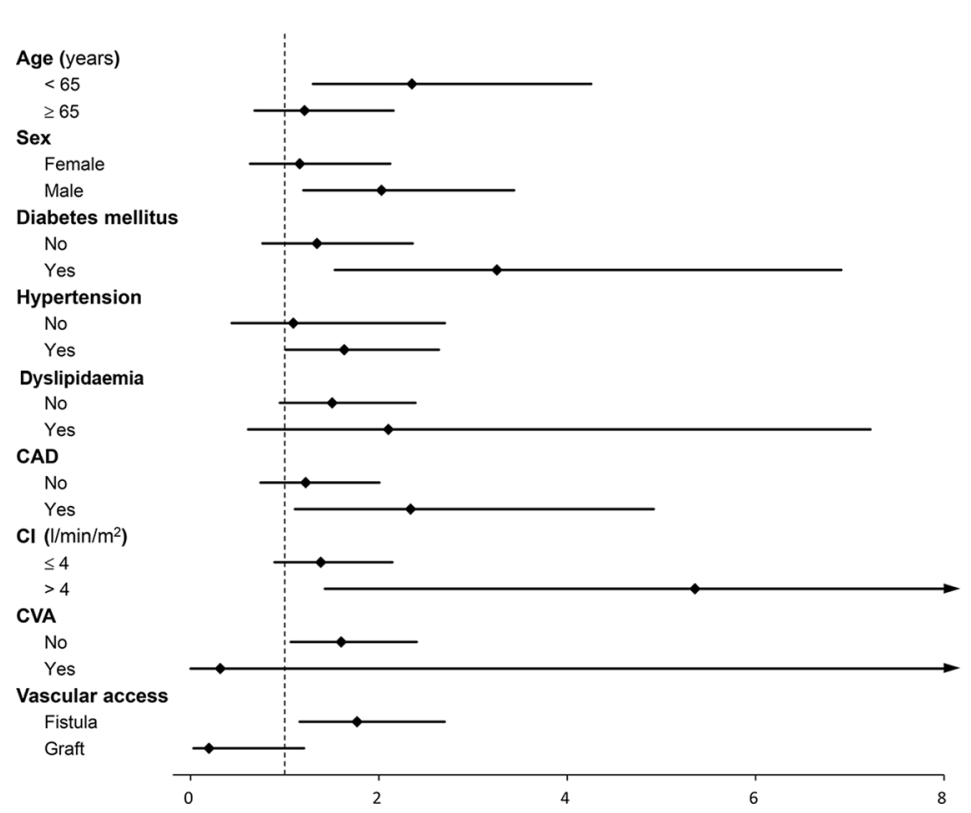

Adjusted HR (Cl95) $\quad P$ value for interaction

$\begin{array}{ll}2.35(1.30-4.25) & <.001 \\ 1.21(0.68-2.16) & .02 \\ 1.16(0.63-2.12) & \\ 2.03(1.20-3.44) & .26 \\ 1.34(0.76-2.36) & \\ 3.25(1.53-6.91) & .75 \\ 1.09(0.44-2.70) & \\ 1.63(1.01-2.64) & .74 \\ 1.50(0.95-2.39) & \\ 2.10(0.61-7.22) & .57 \\ 1.22(0.74-2.01) & \\ 2.34(1.11-4.92) & .01 \\ 1.38(0.89-2.14) & \\ 5.36(1.43-20.10) & .87 \\ 1.60(1.07-2.40) & \\ 0.32(0.001-120.86) & \\ 1.77(1.16-2.70) & \\ 0.20(0.03-1.20) & \end{array}$

Figure 3 Subgroup analysis of the association of lower access flow (Qa $<1000 \mathrm{~mL} / \mathrm{min})$ with all-cause mortality. Each factor was adjusted for all other factors in the multivariate Cox regression model (listed in table 3). CAD, coronary artery disease; Cl, cardiac index; CVA, cerebrovascular accident.

misclassification. Moreover, the optimal cut-off access flow level in patients with an AVF was similar to that in patients with an AVG in our study cohort (online supplementary figure S3).

Although a high Qa level of $>2000 \mathrm{~mL} / \mathrm{min}$ has been revealed to be associated with left ventricular dilation and high-output heart failure, Al-Ghonaim et al reported that they did not observe an increased risk of death with regard to high Qa levels, and they suggested conducting additional studies to confirm the increased risk of

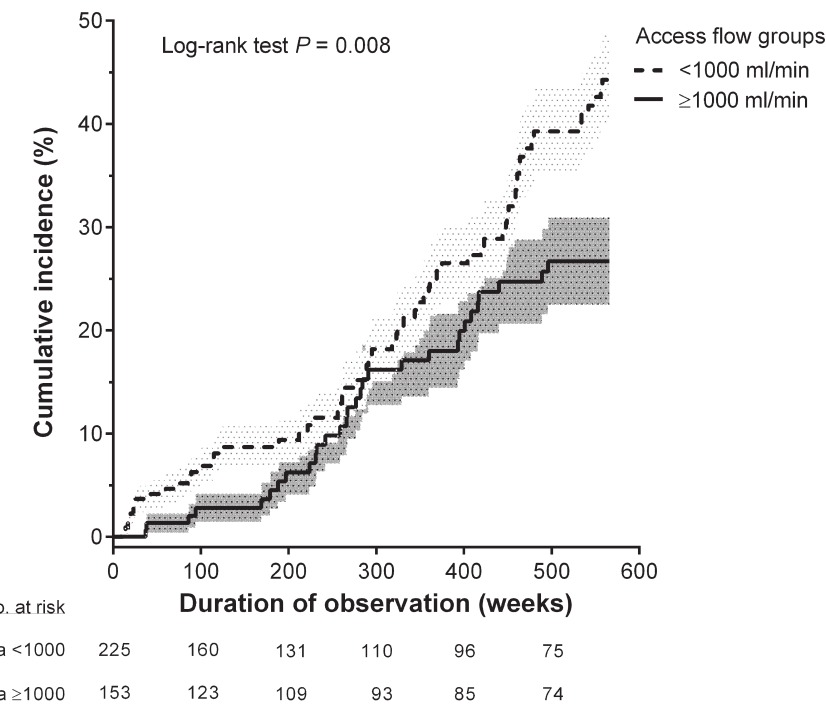

Figure 4 Cumulative incidences (with standard errors) of vascular mortality among the patients in different access flow $(\mathrm{Qa})$ groups. Incidence rate of the composite events of cardiovascular or cerebrovascular death was significantly higher in the lower Qa group $(<1000 \mathrm{~mL} / \mathrm{min})$ than in the higher Qa group ( $\geq 1000 \mathrm{~mL} / \mathrm{min})$ during the follow-up period (log-rank test; $p=0.008$ ). mortality at lower Qa levels. ${ }^{811-13}$ Regarding the Qa level of $<2000 \mathrm{~mL} / \mathrm{min}$, our results suggest that lower Qa levels per se are associated with all-cause mortality in chronic HD patients. Taken together, Qa levels of $1000-2000 \mathrm{~mL} /$ min may be more reasonable for chronic HD patients. However, additional studies are required to confirm our findings.

Studies have reported a positive correlation between $\mathrm{CO}$ and Qa. ${ }^{1325}$ In the current study, Qa moderately and positively correlated with CI, which is in agreement with the findings of a previous study. Kuo et $a t^{26}$ reported a significant association of vascular access dysfunction with subsequent major adverse CV events in chronic HD patients. These findings imply that lower Qa levels may be correlated with vascular access dysfunction, lower cardiac function and subsequent CV or cerebrovascular events. In the current study, the increased risk of vascular mortality was associated with lower Qa levels in the univariate analysis. After adjustment for confounders, the risk of vascular mortality became borderline significant, suggesting that the effect of lower Qa levels on vascular mortality may be mediated through CV risk factors. However, it remains unclear whether poor clearance due to low Qa negatively impacts CI or low CI directly leads to low Qa. In the former case, vascular interventions to augment Qa may improve survival in chronic HD patients, whereas in the latter case, improving Qa might neither improve survival nor be possible without improving CI. Further researches are needed to clarify whether vascular interventions augmenting Qa or improvement of cardiac function while $\mathrm{Qa}<1000 \mathrm{~mL} / \mathrm{min}$ improve survival and other outcomes in these patients.

Subgroup analyses revealed that Qa significantly interacted with age, sex and CI regarding the risk of all-cause 
mortality, suggesting that chronic HD patients with lower Qa levels have a significantly higher risk of all-cause mortality, particularly in those are male, those aged $<65$ years or those with a CI of $>4 \mathrm{~L} / \mathrm{min} / \mathrm{m}^{2}$. Compared with patients aged $<65$ years, those aged $\geq 65$ years may have more comorbidities in addition to being more malnourished; therefore, Qa may have less add-on effects on all-cause mortality in HD patients aged $\geq 65$ years compared with those aged $<65$ years. ${ }^{27}$ In general, vessel sizes are smaller in female HD patients than in male HD patients. ${ }^{28}$ This inherent difference in vascular characteristics between sexes might influence Qa ${ }^{29}$ Female HD patients are more prone to have lower Qa levels than male patients are; therefore, the performance of lower Qa levels in predicting mortality could be attenuated in female HD patients. Moreover, we observed a significantly higher risk of all-cause mortality in patients with a high CI $\left(\mathrm{CI}>4 \mathrm{~L} / \mathrm{min} / \mathrm{m}^{2}\right)$ in the subgroup analyses. A low Qa-to-CO ratio suggests vascular access dysfunction, which leads to morbidity and mortality in HD patients. ${ }^{25} 3031$ Our results suggest that the risk of mortality would be even higher in HD patients having higher cardiac function but lower access flow. Moreover, no significant interactions were observed among the remaining subgroups, including diabetes mellitus, hypertension, dyslipidaemia, CAD, CVA and vascular access type. Additional studies are required to elucidate the effect of Qa on mortality in these subpopulations.

Our study has several limitations that should be addressed. First, Qa and CI were simultaneously measured annually, but Qas were routinely measured trimonthly at our HD unit. In this study, we used baseline Qa measured at entry of the study instead of the average Qa during follow-up; some patients may have been misclassified with respect to different Qa levels, although longitudinal changes in the Qa levels over time may have been relatively small. ${ }^{32}$ Further studies with longitudinal measurements of Qa to clarify the independent role of baseline Qa are needed. Second, variables considered to be associated with mortality, including smoking habits, body mass index and prior use of central venous catheters, were unavailable in our registry data based on the retrospective design. Third, a Qa level changes inevitably during the longterm follow-up because of the development of intimal hyperplasia of vascular access and the following radiological or surgical interventions. In our HD unit, patients with a Qa level of $<600 \mathrm{~mL} / \mathrm{min}$ in grafts and those with a Qa level of $<400-500 \mathrm{~mL} / \mathrm{min}$ in fistulae were routinely referred for fistulography to diagnose stenosis or thrombosis. ${ }^{6}$ Further studies focusing on the impact of vascular interventions are also needed. Radiological or surgical interventions for vascular access dysfunction could influence the relationship between $\mathrm{Qa}$ and mortality. This might explain the lower but insignificant aHR of mortality in our patients with a Qa level of $<500 \mathrm{~mL} / \mathrm{min}$ than in patients with a Qa level of $500-999.9 \mathrm{~mL} / \mathrm{min}$.
Hence, patients with a Qa level of $500-999.9 \mathrm{~mL} / \mathrm{min}$ might have the highest risk of death among the four groups. Therefore, more attention should be focused on these patients to improve their survival. Fourth, changes with the type and location of vascular access in patients during long-term follow-up were not taken into accounts in the cohort study. Finally, as the major population in Taiwan is of Han Chinese ethnicity, our results may not be generalised to other ethnic backgrounds.

In conclusion, our data suggest that a lower Qa level $(<1000 \mathrm{~mL} / \mathrm{min})$ is independently associated with both short-term and long-term all-cause mortality in chronic HD patients. We suggest monitoring Qa levels for achieving early detection of vascular access dysfunction as well as for realising optimal care and reducing mortality. Physicians should be vigilant when the Qa is $<1000 \mathrm{~mL} / \mathrm{min}$ in chronic HD patients.

\section{Author affiliations}

${ }^{1}$ Division of Nephrology, Department of Internal Medicine, Shin-Kong Wu Ho-Su Memorial Hospital, Taipei, Taiwan

${ }^{2}$ School of Medicine, Fu-Jen Catholic University, New Taipei, Taiwan ${ }^{3}$ Institute of Clinical Medicine, National Yang-Ming University, Taipei, Taiwan ${ }^{4}$ Division of Nephrology, Department of Internal Medicine, Changhua Christian Hospital, Changhua, Taiwan

${ }^{5}$ School of Medicine, Chung-Shan Medical University, Taichung, Taiwan

${ }^{6}$ Division of Cardiovascular Surgery, Department of Surgery, Shin Kong Wu Ho-Su Memorial Hospital, Taipei, Taiwan

${ }^{7}$ Internal Medicine Research Center, Changhua Christian Hospital, Changhua, Taiwan ${ }^{8}$ Division of Nephrology, Department of Medicine, Taipei Veterans General Hospital, Taipei, Taiwan

${ }^{9}$ Department and Institute of Physiology, National Yang-Ming University, Taipei, Taiwan

Acknowledgements We are grateful to the assistance of the entire staff at the haemodialysis centre of Shin Kong Wu Ho-Su Memorial Hospital.

Contributors All authors reviewed the manuscript. C-KW collected and interpreted the data and wrote the manuscript. C-LW and C-TK ran the data, performed statistical analyses and helped to write the manuscript. C-KW and C-LW determined the concept and design of this study. C-KW, C-LW, C-HL, J-GL and D-CT contributed to the discussion and manuscript revision. D-CT and C-LW conceived the study and are the guarantors of this publication.

Funding This study was supported by grant SKH-8302-102-DR-05 from the ShinKong Wu Ho-Su Memorial Hospital Research Foundation, the Novel Bioengineering and Technological Approaches to Solve Two Major Health Problems in Taiwan sponsored by the Taiwan Ministry of Science and Technology Academic Excellence Program (MOST 105-2633-B-009-003), and Foundation for Poison Control.

Competing interests None declared.

Patient consent Detail has been removed from this case description/these case descriptions to ensure anonymity. The editors and reviewers have seen the detailed information available and are satisfied that the information backs up the case the authors are making.

Ethics approval The study was approved by the institutional review board of Shin Kong Wu Ho-Su Memorial Hospital (approval number 20160104R).

Provenance and peer review Not commissioned; externally peer reviewed. Data sharing statement № additional data are available.

Open Access This is an Open Access article distributed in accordance with the Creative Commons Attribution Non Commercial (CC BY-NC 4.0) license, which permits others to distribute, remix, adapt, build upon this work non-commercially, and license their derivative works on different terms, provided the original work is properly cited and the use is non-commercial. See: http://creativecommons.org/ licenses/by-nc/4.0/ 
(c) Article author(s) (or their employer(s) unless otherwise stated in the text of the article) 2017. All rights reserved. No commercial use is permitted unless otherwise expressly granted.

\section{REFERENCES}

1. Coentrão L, Turmel-Rodrigues L. Monitoring dialysis arteriovenous fistulae: it's in our hands. J Vasc Access 2013;14:209-15.

2. Hakim RM, Breyer J, Ismail N, et al. Effects of dose of dialysis on morbidity and mortality. Am J Kidney Dis 1994;23:661-9.

3. Rocco MV, Bleyer AJ, Burkart JM. Utilization of inpatient and outpatient resources for the management of hemodialysis access complications. Am J Kidney Dis 1996;28:250-6.

4. Vascular Access 2006 Work Group. Clinical practice guidelines for vascular access. Am J Kidney Dis 2006;48(Suppl 1):S176-247.

5. NKF-DOQI clinical practice guidelines for vascular access. Nationa kidney foundation-dialysis outcomes quality initiative. Am J Kidney Dis 1997;30(4 Suppl 3):S150-91.

6. Vascular Access Work Group. Clinical practice guidelines for vascular access. Am J Kidney Dis 2006;48(Suppl 1):S248-73.

7. Besarab A. Advances in end-stage renal diseases 2000. Access monitoring methods. Blood Purif 2000;18:255-9.

8. Al-Ghonaim M, Manns BJ, Hirsch DJ, et al. Relation between access blood flow and mortality in chronic hemodialysis patients. Clin J Am Soc Nephrol 2008;3:387-91.

9. Coresh J, Longenecker JC, Miller ER, et al. Epidemiology of cardiovascular risk factors in chronic renal disease. J Am Soc Nephrol 1998;9(12 Suppl):S24-30.

10. Lowrie EG, Laird NM, Parker TF, et al. Effect of the hemodialysis prescription of patient morbidity: report from the National Cooperative Dialysis Study. N Engl J Med 1981;305:1176-81.

11. MacRae JM, Pandeya S, Humen DP, et al. Arteriovenous fistulaassociated high-output cardiac failure: a review of mechanisms. Am J Kidney Dis 2004;43:e17-22.

12. Bailey WB, Talley JD. High-output cardiac failure related to hemodialysis arteriovenous fistula. J Ark Med Soc 2000;96:340-1.

13. Basile $\mathrm{C}$, Lomonte $\mathrm{C}$, Vernaglione $\mathrm{L}$, et al. The relationship between the flow of arteriovenous fistula and cardiac output in haemodialysis patients. Nephrol Dial Transplant 2008;23:282-7.

14. Gotch FA, Sargent JA. A mechanistic analysis of the National Cooperative Dialysis Study (NCDS). Kidney Int 1985;28:526-34.

15. Krivitski NM. Novel method to measure access flow during hemodialysis by ultrasound velocity dilution technique. Asaio $\mathrm{J}$ 1995;41:M741-5.

16. Lopot F, Nejedlý B, Sulková S, et al. Comparison of different techniques of hemodialysis vascular access flow evaluation. Int $J$ Artif Organs 2003;26:1056-63.
17. Garland JS, Moist LM, Lindsay RM. Are hemodialysis access flow measurements by ultrasound dilution the standard of care for access surveillance? Adv Ren Replace Ther 2002;9:91-8.

18. Lok CE, Bhola $\mathrm{C}$, Croxford $\mathrm{R}$, et al. Reducing vascular access morbidity: a comparative trial of two vascular access monitoring strategies. Nephrol Dial Transplant 2003;18:1174-80.

19. Heagerty PJ, Zheng Y. Survival model predictive accuracy and ROC curves. Biometrics 2005;61:92-105.

20. Collins AJ, Foley RN, Herzog C, et al. Excerpts from the US renal data system 2009 annual data report. Am J Kidney Dis 2010;55(1 Suppl 1):A6-A7.

21. Cooper BA, Penne EL, Bartlett LH, et al. Protein malnutrition and hypoalbuminemia as predictors of vascular events and mortality in ESRD. Am J Kidney Dis 2004;43:61-6.

22. Hwang JC, Wang CT, Chen CA, et al. Hypokalemia is associated with increased mortality rate in chronic hemodialysis patients. Blood Purif 2011;32:254-61.

23. Hamano T. [Serum phosphate level and the prognosis of dialysis patients]. Clin Calcium 2009;19:174-9.

24. Jean G, Lataillade D, Genet L, et al. Association between very low PTH levels and poor survival rates in haemodialysis patients: results from the French ARNOS cohort. Nephron Clin Pract 2011;118:c211-6.

25. Pandeya S, Lindsay RM. The relationship between cardiac output and access flow during hemodialysis. Asaio J 1999;45:135-8.

26. Kuo TH, Tseng CT, Lin WH, et al. Association between vascular access dysfunction and subsequent major adverse cardiovascular events in patients on hemodialysis: a Population-Based Nested Case-Control Study. Medicine 2015;94:e1032.

27. Canaud B, Tong L, Tentori F, et al. Clinical practices and outcomes in elderly hemodialysis patients: results from the Dialysis Outcomes and Practice Patterns Study (DOPPS). Clin J Am Soc Nephrol 2011;6:1651-62.

28. Dodge JT, Brown BG, Bolson EL, et al. Lumen diameter of normal human coronary arteries. Influence of age, sex, anatomic variation, and left ventricular hypertrophy or dilation. Circulation 1992;86:232-46.

29. Marcus RJ, Marcus DA, Sureshkumar KK, et al. Gender differences in vascular access in hemodialysis patients in the United States: developing strategies for improving access outcome. Gend Med 2007;4:193-204.

30. Feldman HI, Kobrin S, Wasserstein A. Hemodialysis vascular access morbidity. J Am Soc Nephrol 1996;7:523-35.

31. Dhingra RK, Young EW, Hulbert-Shearon TE, et al. Type of vascular access and mortality in U.S. hemodialysis patients. Kidney Int 2001;60:1443-51.

32. Begin V, Ethier J, Dumont M, et al. Prospective evaluation of the intra-access flow of recently created native arteriovenous fistulae. Am J Kidney Dis 2002;40:1277-82. 